guessing. With a little care and application, the technique can tailor the approach to a paper so as to maximise the likelihood of success.

\section{Scientific method}

Up to the stage of university entrance, the British educational system has in the past relied on multiple choice examinations where negative marking is not used. It is thus advantageous to answer all questions, going through a paper over and over again, answering progressively harder questions. The method described here has evolved from this approach.

This is a form of audit of personal performance. Take a test paper. Consider where you feel the pass mark is likely to be, for example, $55 \%$. Answer only the questions of which you are absolutely sure. If the number of questions answered is $55 \%$ or more, stop. Repeat the paper, answering all the questions. If you now mark the paper, the results will indicate how well you have done, answering the above proportions of questions.

The above procedure can be repeated, answering various proportions, e.g. $70 \%, 80 \%$ and $90 \%$. There is an obvious problem in deciding which questions to answer and which to omit. Make notes on the examination paper. If you answer all the questions you are positive about first, marking those you are fairly certain of, then you can work over the paper again.

Repeating this procedure with a number of papers will allow you to plot a graph of:

Percentage Answered against Mark Scored.

This should quickly allow you to determine the percentage of questions to answer in order to achieve your best scoring chance. Obviously this will differ from person to person, but a realistic result might be $80 \%$ of questions to be answered. Armed with this information, you could reasonably approach an examination by going through the paper until your target percentage of questions has been answered, stopping and leaving.

\section{Conclusions}

Examinations are serious affairs and deserve careful strategic planning to try and eliminate bad fortune. Confidence is paramount, and simple strategies build confidence. There are two caveats. Firstly if your practice scoring is uniformly low, then sadly it is probable that more knowledge is required. Secondly, if you go through the examination paper for the first time, answering all you are positive of and you confidently reach your target quota, you should of course complete the remaining answers, but should not run through the paper again.

Finally, this approach may not be to everyone's taste. It seems to have test-retest reliability but there are obviously problems in demonstrating inter-rater reliability. As with statistical analyses, there may be assumptions in generalising from a small sample. Nevertheless, the above concept may be a valuable option for those who are prepared to experiment and open to logical argument.

\section{Acknowledgements}

I am grateful to Dr Greg Wilkinson for his comments.

\section{References}

GLEw, G. (1981) Multiple Choice Questions in Psychiatry, Second edition. London: Butterworths.

Grant, C., McDonald, G. \& Bell, P. (1986) Multiple Choice Questions. Hemel Hempstead: Pastest Service. Levi, M. (1990) MCQs for the MRCPsych Part 1. Lancaster: MTP Press.

LEWIS, A. (1958) Between guesswork and certainty in psychiatry. Lancet, 1, 171-5.

(1958) Between guesswork and certainty in psychiatry. Lancet, 1, 227-30.

\title{
MCQs: a suggested study technique
}

\author{
Marcellino G. Smyth, Senior Registrar, Uffculme Clinic, Mosely, \\ Birmingham B13 8QD
}

The MCQ has now become firmly established as an integral part of post-graduate psychiatric examinations. In my experience a number of trainees have particular difficulty with MCQ tests such that they find them threatening, and in some cases a repeated stumbling block to the advancement of their careers.
A minority within this group may consider the MCQ test to lie in the realm of the arcane and mysterious and find themselves subject to repeated worry and frustration. While advice on techniques for answering is plentiful (sample textbooks and manuals on examination technique) I am not aware that there has 
been any focus to date on study technique. I would like to suggest a personal approach for MCQ study (based on successful application) which I believe fully integrates MCQ with general psychiatric study and emphasises the potential of 'MCQ thinking' in refining ones overall psychiatric knowledge.

\section{$M C Q$ consideration time}

The maxim "if you aim at nothing you will surely hit it" applies to MCQ tests as readily as most endeavours. Those candidates with repeated difficulty in MCQs may rely on general reading and study, and hope that they can apply this successfully when faced with the MCQ sections. The resulting mismatch between study and examination can be problematic. The key to better performance lies in MCQ awareness. This involves the incorporation of MCQ 'consideration time' in studying any topic. Time spent in examining text or literature material with this aim should pay dividends in terms of easier recognition of MCQs in examinations. It is important to state that this does not mean simply an exercise in obsessionally reading the small print or in concentrating on the minutiae of topics. MCQ consideration time is usefully applied at a number of conceptual levels.

In this MCQ study, the discipline of distinguishing between fact and opinion is essential. Here, the search is for the 'controversy' factor inherent in any body of information. Part of MCQ consideration time involves organising data along confidence intervals. For instance, in considering the factors reported to increase the risk of tardive dyskinesia, students might ask themselves the question: 'How dogmatic am I prepared to be about these factors in terms of MCQ formulation?' They might conclude (from reading and balancing the views of different texts) that they could confidently assert old age, female sex, and brain injury to be accepted risk factors, but that more controversy surrounded the issue of high dose neuroleptics. By such familiarity, any dogmatic statement incriminating high dose neuroleptics is likely to be false or requiring a more cautious approach.

The search becomes an exercise in differentiation between hard (agreed), and soft (controversial) data. Most subjects incorporate a welcome plethora of 'hard', high factual content information. These data can be just as welcome to the student as to the setter of an MCQ exam. Put another way, the student can look out for information which an MCQ setter would find 'user friendly'. Examples are neuroanatomy, receptor subtypes, pathological changes, neuroendocrinology, tables, figures and percentages (incidence, age of onset, prognosis, genetic susceptibility etc.). In less clearly factual areas (sociology, expressed emotion, family influences in the aetiology of schizo- phrenia) the search for what constitutes a fact, continues. One might look at Litz's theories and generate the following statements in progressively increasing level of sophistication; his theory revolved around atypical dominance patterns (T), skew involves one parent being dominant while the other is inadequate (T) schism and skew often occur in the same family (F). His work has been well replicated (F). He tried to experimentally validate his theories $(F)$.

This exercise is usefully integrated with general study and critical analysis in that interesting and stimulating questions arise which can spur the student to wider reading.

\section{Transpositions}

Having identified, considered and categorised the 'factual' elements relevant to a topic, the next stage is to develop transpositions. Examination questions will seldom be phrased similar to the source text, hence a need arises to expand items of knowledge as a basis for better appreciation, recall, and MCQ recognition. The following are suggested transpositions for different items:

Basic fact: Adverse prognostic factors in depression of old age are a late onset of depression, concurrent cerebral or non-cerebral organic disease, a senile habitus, continuous depression for two years.

Transpositions: Depression in the elderly is unfavourable affected by: onset immediately post retirement (F), diabetes mellitus ( $T$ ), appearance older than age (T), unremittant depression for over 18 months (T).

Basic fact: Neurological involvement in Friedrich's ataxia includes nystagmus, cerebellar, pyramidal and posterior column damage.

Transpositions: In Friedrich's ataxia the following are recognised; Facilis Gracilis affected $(\mathrm{T})$, absence of tendon reflexes $(T)$, extensor plantar response $(T)$, intact vibration sense $(F)$, pes cavus $(T)$. The exercise of developing transpositions can be particularly useful for those for whom English is not a first language.

\section{Near misses}

MCQ tests incorporate an emphasis on precise terminology. In devising one's own questions relevant to any topic, students should be alert to such precision, so that they might more easily recognise 'near misses'. Examples are: catalepsy/cataplexy, 5-hydroxy tryptophan/5-hydroxy tryptamine, coprophagia/copralalia, echolalia/echopraxia, erectile impotence/ retarded ejaculation, etc. Spotting near misses in the exam can greatly improve one's confidence that the statement is false (depending on the phraseology). 


\section{Depth}

While it is obvious that every interested student will endeavour to increase their depth of knowledge, I think this has particular relevance to MCQ study. A guiding principle is that it is more difficult to judge an unrecognisable statement as false, than to assert a recognisable one as true. In my opinion, this is the most fundamental truth underlying the composition of MCQ tests. The reason for this lies in the limited or finite quanta of knowledge known about any topic, as compared to the infinite number of false statements that may be proposed. Thus, there are only so many documented side effects of tricyclic drugs, or clinical features of Klinefelter's syndrome, but limitless opportunity to pose untrue or non-documented side effects or clinical features respectively. When faced with a statement or association which the student has not seen or considered before, the more depth of knowledge he can recruit in that area, the more confident can he be in asserting the falsehood of untrue propositions. Increased depth of familiarity should reduce the recurrent dilemma in the exam when faced with a novel association of "Well, I have not seen this before but it looks quite possible!" As the depth of the exam is often set at the level of reference papers to major topics, reading the reference articles from one's major textbook is the most direct route to harmonising one's study and the exam itself.

\section{Conclusion}

MCQ tests focus on the evaluation of propositional statements. Ongoing evaluation of knowledge in MCQ terms as a formal discipline can be both a stimulating adjunct to general study and can improve exam performance.

\title{
The psychiatric MCQ: are 'possibles' always true?
}

\author{
Jonathan I. Bisson, Registrar, Queen Elizabeth Military Hospital, London SE18 4JR
}

\author{
"Depression of mood may be caused by \\ Propranolol \\ Reserpine \\ Clonidine \\ Frusemide \\ Digoxin"
}

The above specimen MRCPsych Part 1 question (Royal College of Psychiatrists, 1989) illustrates well the difficulties facing the examination candidate who is required to make a TRUE/FALSE/DON'T KNOW decision in the knowledge that an incorrect response will result in a negative mark.

The term "may be" in this question is problematic. Holden's (1987) useful book on examination techniques states " 'may be' means that it is recognised (i.e. reported) to occur." "May be" appears to imply a possibility, however remote. Therefore the prudent candidate is likely to answer TRUE to the vast majority of such questions assuming that most things are possible. But frusemide and digoxin? Surely not?

The sources of information open to the average candidate produce a variety of answers to the original question.

The Oxford Textbook of Psychiatry (Gelder et al, 1988) states that diuretics may cause depression due to electrolyte depletion, that digitalis may cause mood disturbance and that both reserpine and sympathetic blockers may cause depression. No mention is made of clonidine. This information allows us to answer the question TT?TT.

The Association of the British Pharmaceutical Industry Data Sheet Compendium 1989-90 states that depression is a possible side effect with clonidine and a possible toxic effect of digoxin. Depression is not among the possible side effects listed of propranolol and frusimide. Reserpine has no entry. Our answer from this source is ??T? or F?TFT if we assume the adverse effects listed to be exhaustive.

The British National Formulary (1990) provides a ?TT?? answer or FTTFF answer. Also stated is the fact that "any drug MAY produce unwanted or unexpected adverse reactions" suggesting a TTTTT response is perhaps correct.

Other sources of information, including journals, throw further light on to the question. Reserpine, which acts as an antihypertensive by depleting tissue stores of monoamines, has long been suspected of causing depression by the same mechanism. Similarly, clonidine decreases monoamine availability at the synapse, exerting its effect through pre-synaptic alpha 2 adrenoceptor agonism. It may cause depression through the same mechanism.

Propranolol and other beta blockers have been associated with depression in several studies. For example, Avorn et al (1986) showed that $23 \%$ of a large population taking beta blockers for two years were prescribed a tricyclic antidepressant in that 